\title{
Liquid Sheet characteristics of Two Impinging Jets with Different
}

\section{Diameter}

\author{
Sina Kashanj ${ }^{1}$, Yeganeh Saffar ${ }^{2}$, Azadeh Kebriaee*1 \\ ${ }^{1}$ Aerospace Engineering, Sharif University of Technology, Tehran, Iran \\ ${ }^{2}$ Aerospace Engineering, Amirkabir University of Technology, Tehran, Iran \\ ${ }^{*}$ Corresponding author email: Kebriaee@Sharif.edu
}

\begin{abstract}
In this paper, the liquid sheet properties of two impinging jets with different diameter are investigated with the Reynolds number of jets $R e_{j}$ ranging from 81 to 243 . Shadowgraph imaging and image processing have been applied to capture the liquid sheet and analyse its properties. Results show that two impinging jets with different diameter but with the same velocity of the jets forms a stable and laminar liquid sheet. However, the liquid sheet of two jets with the different diameter but with the same momentum of the jets is unstable fragmenting liquid ligaments and droplets in a specific diameter difference criterion.
\end{abstract}

\section{Keywords}

Impinging two jets, Liquid Sheet, Shadowgraph imaging, Asymmetric conditions

\section{Introduction}

Impinging liquid jets is a technique for atomizing and mixing the liquids for different applications [1]. In the aerospace industry, it is used for atomization of the fuel and oxidizer liquids [2]. This technique can be applied for atomization of the high viscosity and nonNewtonian liquids such as the gelled fuels because of its simplicity and high-performance mixing and atomization [3]. In chemical reactors, impinging of two jets can be used in crystallization and precipitation process [4], [5], synthesis of silver nanoparticles [6], and forming clathrate hydrates [7]. Impinging jets also can be developed with the $\mathrm{UV} / \mathrm{H}_{2} \mathrm{O}_{2}$ reactors for wastewater treatment [8].

When two liquid jets impinge, a liquid sheet forms perpendicular to the axis of the two jets [2]. The flow growth radially from the impinging point and it restrained by the surface tension force of the liquid in the opposite direction of the inertia force which leads to the formation of the thick liquid rim at the boundary of the liquid sheet [9]. The liquid flows from the top of the liquid rim to the down and the flow of the liquid rim from the left hand side collides to the right hand side rim-flow [9]. This collision of the liquid rims acts like the impact of the two jets and produces the secondary liquid sheet and rim. Same procedure for the second liquid sheet can make the third liquid sheet [10]. In case of higher Reynolds number of the jets, the secondary liquid sheet is unstable which leads to formation of ligaments and droplets [10]. The same instability can happen in higher Reynolds numbers for the first liquid sheet [10].

Different sources may be responsible for the instability of the liquid sheet. In the impinging two like jets, for the Weber numbers, $W e_{j}=\frac{\rho V_{j}^{2} D_{j}}{\sigma}$, lower than 2000 and higher than 500, Taylor waves are responsible for the instability of the liquid sheet [11]. For Weber numbers higher than 2000 , instability of the liquid sheet has been corresponded to Kelvin-Helmholtz instability [11].

Impinging two jets with low Weber number forms the leaf shaped stable liquid sheet. Specific circumstances such as interaction with an outer object [12], imperfect impact of the two jets [13], and existence of differential in velocity of the two jets [14] can lead to perturbation and 
atomization of the liquid sheet. For specific range of the velocity differential, $\lambda=\left|\frac{V_{j 2}-V_{j 1}}{V_{j 1}}\right|$ the periodic atomization of the liquid sheet known as fishbone regime has been observed [10]. The periodic atomization of the liquid sheet also has been observed in the liquid sheet of the two jets with imperfect impingement in specific range of skewness defined as $\boldsymbol{\delta}=\left|\frac{\boldsymbol{y}\left(\boldsymbol{D}_{\boldsymbol{j} 2}\right)-\boldsymbol{y}\left(\boldsymbol{D}_{\boldsymbol{j} 1}\right)}{\boldsymbol{D}_{\boldsymbol{j}}}\right|$ which is another source of perturbation [14].

In this paper, the liquid sheet properties of the two impinging jets with different diameters for very low Reynolds number of the jets, $81 \leq R e_{j} \leq 243$ has been studied experimentally. The aim of the conducted experiments is to investigate the liquid sheet properties in two different flow conditions of impinging two jets with the same velocity and impinging two jets with the same momentum.

\section{Material and Methods}

Figure 1 illustrates the schematic of the fluid system designed and made for feeding liquid to the nozzles for producing the liquid jets. In this closed-loop fluid system, a centrifugal pump used for pumping the liquid from the liquid tank to the diaphragm of the high-pressure vessel. After charging the diaphragm of the high-pressure vessel, the pressure is added by using an $\mathrm{N}_{2}$ gas cylinder. The connection of the $\mathrm{N}_{2}$ gas cylinder to the vessel will be regulated using a pressure regulator to keep the pressure of the vessel constant during the flow feeding to the nozzles. Two flow meters with a precision of $0.2 \mathrm{ml} / \mathrm{s}$ and two needle valves were used for measuring and controlling the velocity of each jet.

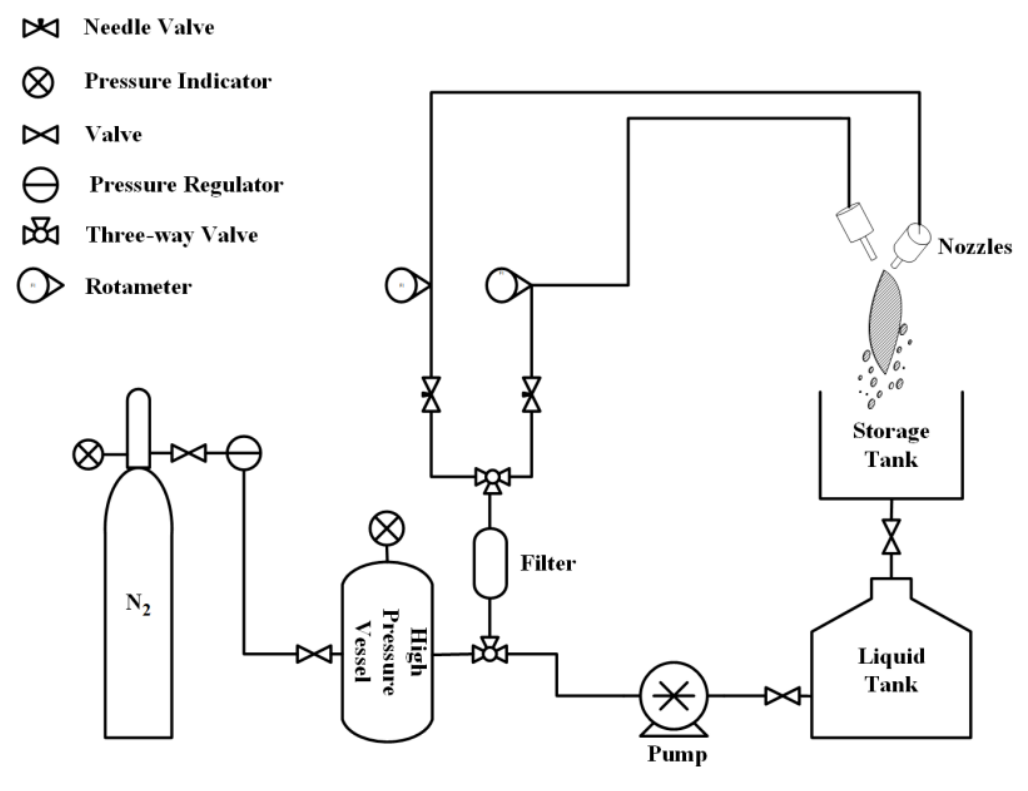

Figure 1. Schematic of the configuration of the nozzles and imaging system

Configuration of the imaging system and nozzles is depicted in Figure 2. Each nozzle was installed to a rotating stage with the precision of $1^{\circ}$ and two jets impinge with an impinging angle $2 \theta$, of $90^{\circ}$. Hypodermic needles were used as the nozzles for producing the liquid jets. For impinging two identical jets, nozzle with an inner diameter, $D_{j}$, of $0.5 \mathrm{~mm}$ has been used. For studying the effect of the impinging of two jets with different diameter, three other nozzles with diameter, $D_{j}$, of $0.6,0.7$, and $0.8 \mathrm{~mm}$ were used as the second nozzles working with the $0.5 \mathrm{~mm}$ nozzle. The difference between the diameter of the two jets is defined as the diameter 
difference factor, $\varepsilon=\frac{\left|\boldsymbol{D}_{j 2}-\boldsymbol{D}_{\boldsymbol{j} 1}\right|}{\boldsymbol{D}_{\boldsymbol{j} 1}}$. The effect of the diameter difference has been studied for the diameter difference factor, $\varepsilon$ of $0,0.2,0.4$, and 0.6 . Two micro-stages with the precision of 10 $\mu \mathrm{m}$ were installed for controlling the position of the first nozzle in $x$ and $z$ directions. In the case of impinging two jets with the same diameter, controlling the position of the first jet can be applied to make the collision of the two jets perfect without any skewness. In the case of impinging two jets with different diameter, the position of the smaller jet is set to be concentric with the second jet.

Shadowgraph technique has been applied for capturing the liquid sheet. A high speed CMOS camera (ximea-MC031CG-SY) has been synced with a high power pulsed LED light source (Photo Freezer v2.5) with the pulse width down to $125 \mathrm{nsec}$ and frequency up to $1000 \mathrm{~Hz}$ to freeze and capture the liquid sheet and droplets. A lens (Navitar 1-19558 2/3") with a focal length of $50 \mathrm{~mm}$ mounted to the camera to capture the field of view, FOV of $50 \times 50 \mathrm{~mm}^{2}$.

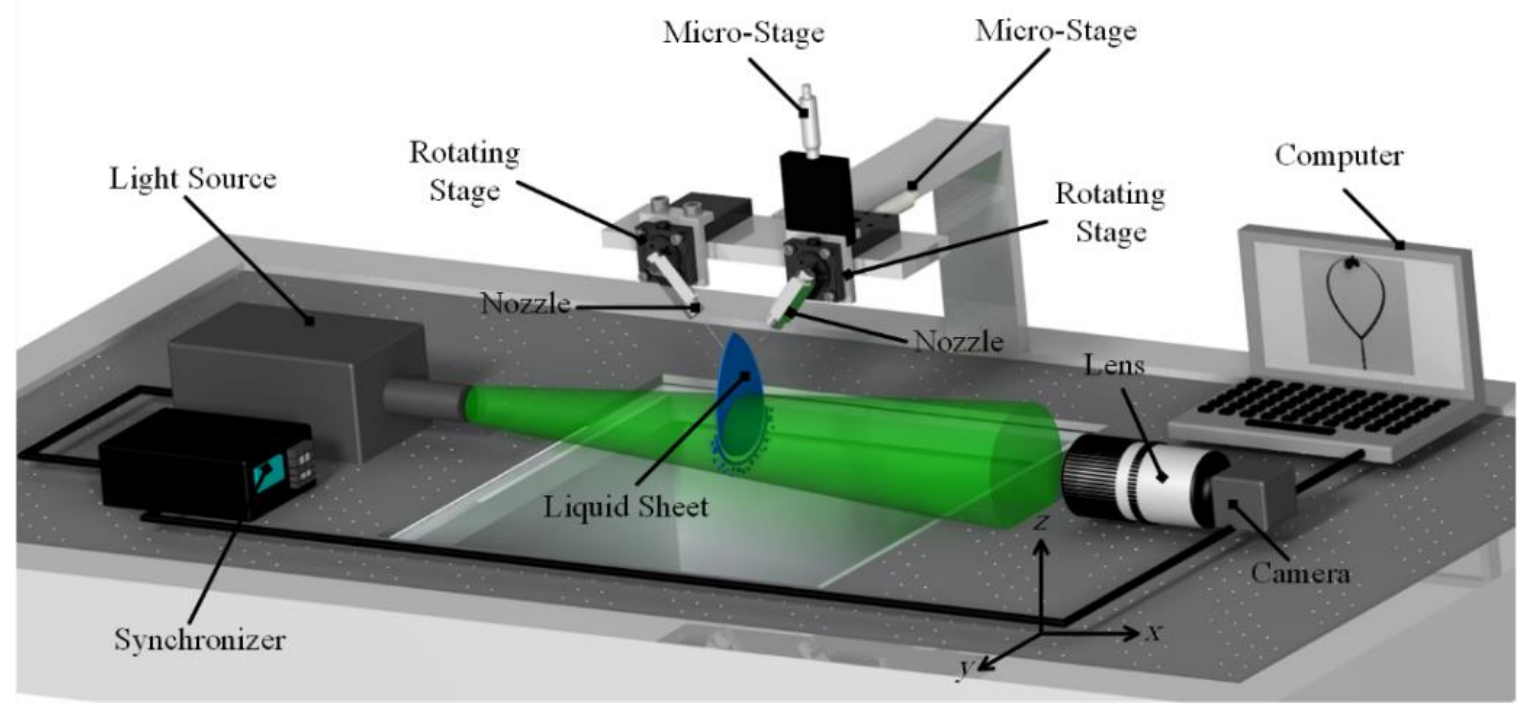

Figure 2. Schematic of the configuration of the nozzles and imaging system

\section{Results and Discussion}

For impinging two jets with different diameters, two possible flow conditions could be assumed, impinging two jets with equal flow rate, momentum, and velocity.

In impinging two jets with the same momentum, $\frac{\boldsymbol{V}_{\boldsymbol{j} 1}}{\boldsymbol{V}_{\boldsymbol{j} 2}}=\frac{\boldsymbol{D}_{\boldsymbol{j} 2}}{\boldsymbol{D}_{\boldsymbol{j} 1}}$ for $\varepsilon>0.2$ the velocity difference coefficient is $\lambda \geq 0.16$. For this range of the velocity coefficient and $81 \leq R e_{j} \leq 243$, the liquid sheet is shown in Figure 3 (left). As shown, the liquid sheet for $\varepsilon=0$ is a stable liquid sheet growing with increasing Reynolds number of the jets. In $R e_{j}=81$, the regime of the liquid sheet is multi-chain regime for all the diameter difference coefficients (Figure 3 (left) (a), (e), (i), and $(\mathrm{m}))$.

In $R e_{j}=162$, the liquid sheet is unstable in $\varepsilon=0.2$ (Figure 3 (left) (f)). The liquid sheet is in a fishbone regime which is an oscillating liquid sheet producing monodisperse droplets due to fragmentation of the liquid rim [12]. 


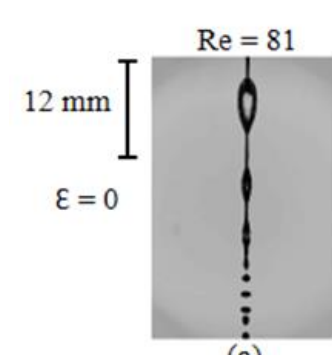

(a)

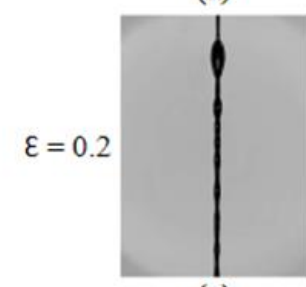

(e)

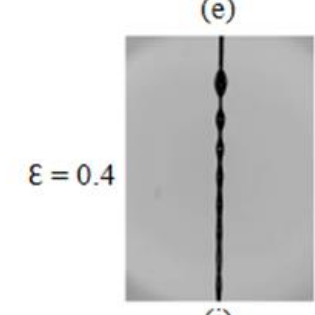

(i)

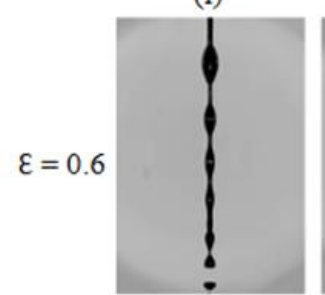

(m)

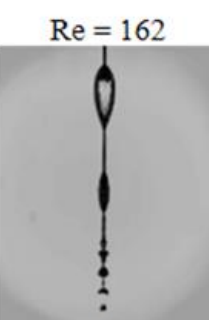

(b)

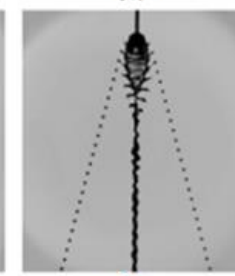

(f)
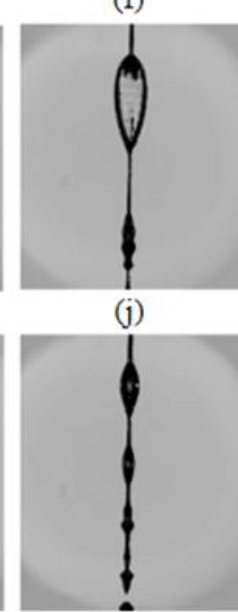

(n)

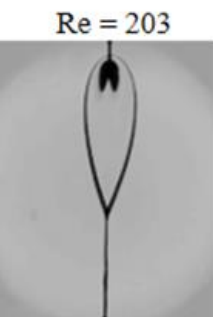

(c)

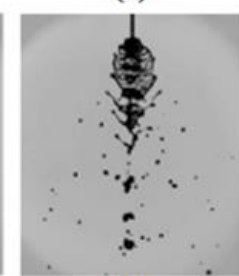

(g)

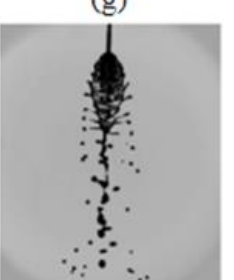

(k)

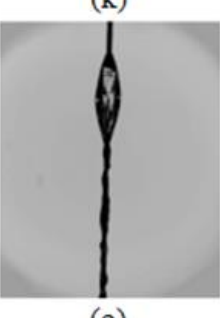

(o)
$\mathrm{Re}=243$

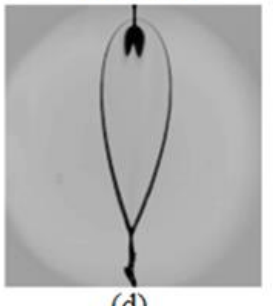

(d)

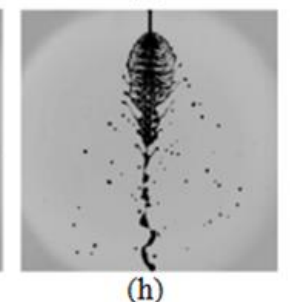

(h)

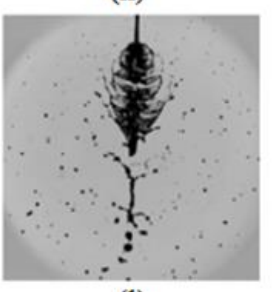

(1)

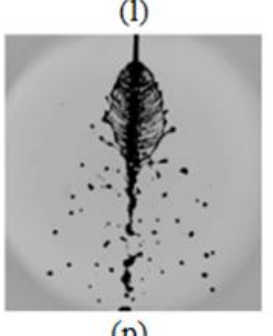

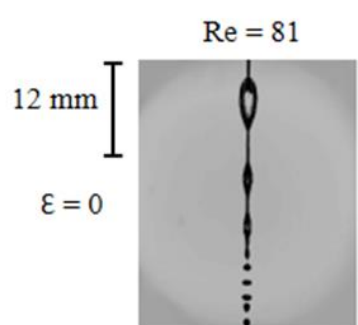

(a)

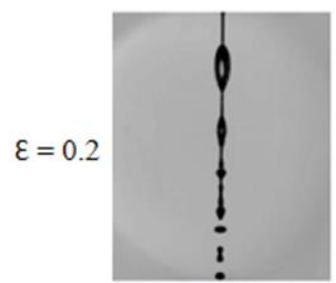

(e)
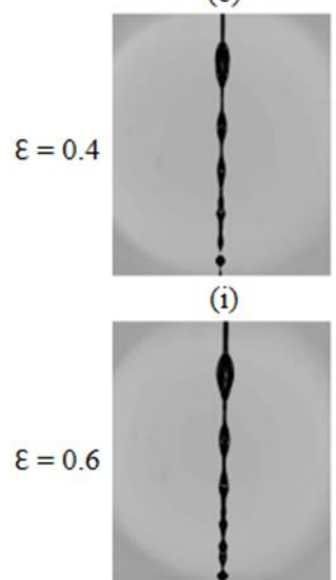

(m)
$\mathrm{Re}=162$

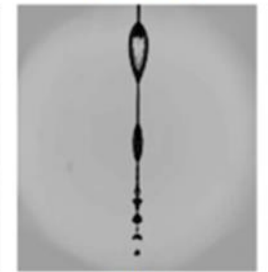

(b)
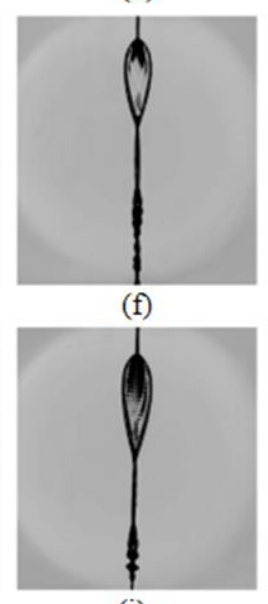

(j)

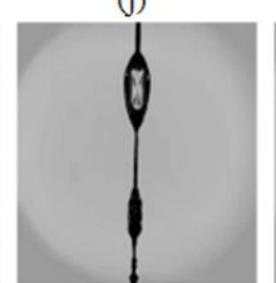

(n)
$\mathrm{Re}=203$

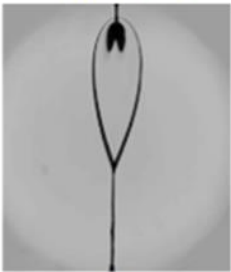

(c)

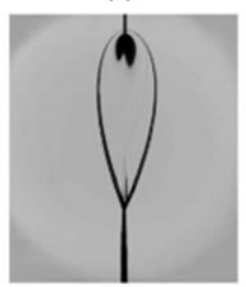

(g)

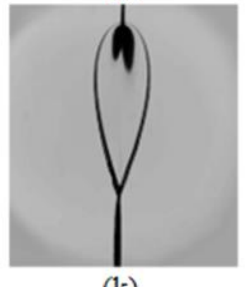

(k)

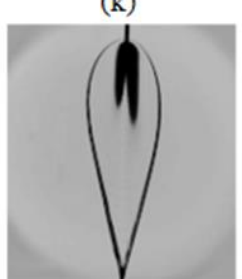

(o)

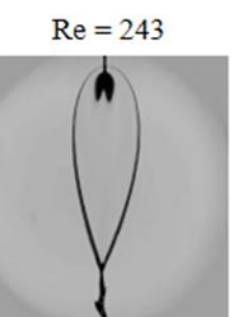

(d)

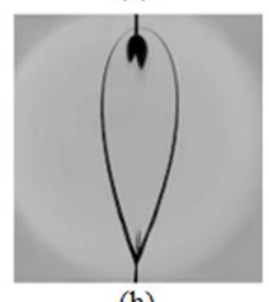

(h)

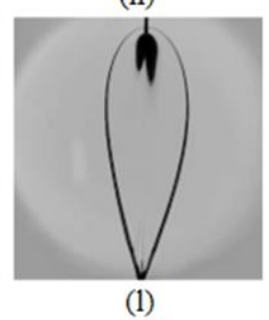

(1)

Figure 3. Liquid sheet of two impinging jets formed by impinging two jets with the same momentum (left) and with the same velocity (right). (a-d) Diameter difference coefficient $\varepsilon=0$, with $R e_{j}$ equal to $81,162,203$, and 243 respectively. (e-h) $\varepsilon=0.2, R e_{j}=81,162,203$, and 243. (i-l) $\varepsilon=0.4, R e_{j}=81,162,203$, and 243 . (m-p) $\varepsilon=0.6$, $R e_{j}=81,162,203$, and 243
$\varepsilon$ 
In the same diameter difference coefficient $\varepsilon$, and for the higher Reynolds number, $R e_{j}$ the liquid sheet is in an unstable regime generating droplets due to fragmentation of both liquid rim and liquid sheet (Figure 3 (left) (g), (h)). In $\varepsilon=0.4$, the instability of the liquid sheet occurs in higher Reynolds number, $R e_{j}=203$ (Figure 3 (left) (k)). Following the same trend, in $\varepsilon=0.6$, the instability of the liquid sheet occurs in a higher Reynolds number, $R e_{j}=243$ (Figure 3 (left) (p)). This shows that the small amount of the difference in diameter of the two jets leads to instability of the liquid sheet even in low Reynolds numbers. It means the Reynolds number in which the liquid sheet starts to be unstable increased by increasing the diameter difference coefficient, $\varepsilon$.

Figure 4 shows the variations of the liquid sheet length and width with the diameter difference coefficient $\varepsilon$ for four Reynold numbers. The criterion in which the length and width of the liquid sheet is identified illustrated in Figure 5 . As can be expected, it is observed that both length and width of the liquid sheet increase by increasing the Reynolds number. In this figure, the square around the points show that the liquid sheet of that specific point is laminar and the circle shows that the liquid sheet in that point is unstable fragmenting ligaments and droplets.

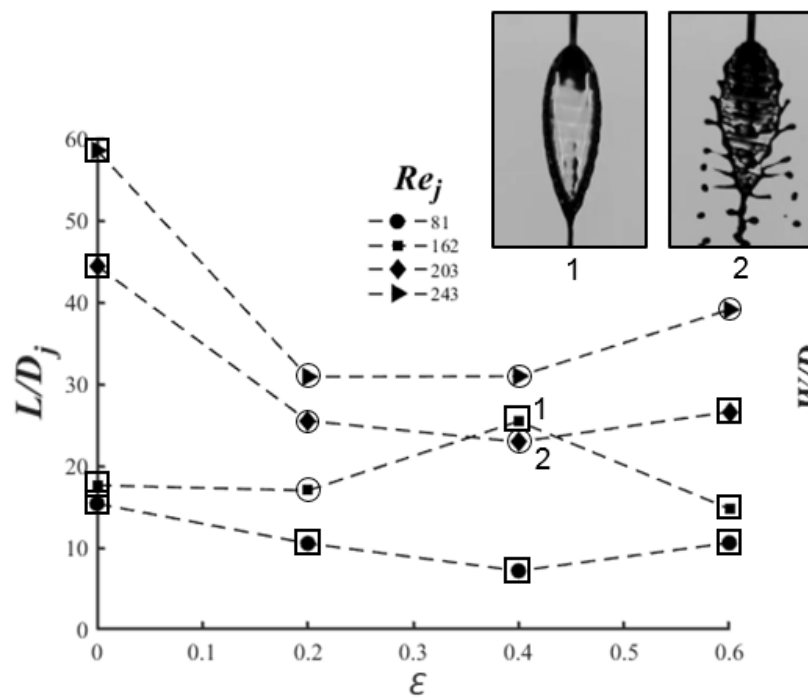

(a)

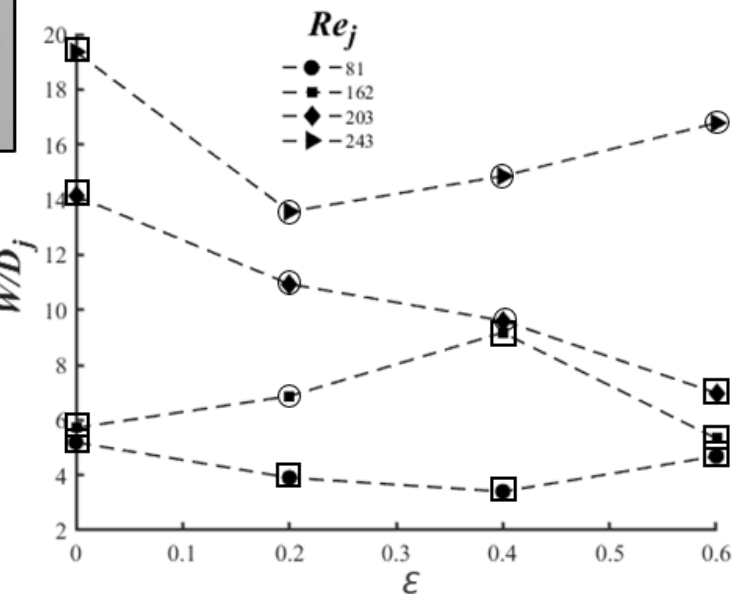

(b)

Figure 4. Variation of the (a) length and (b) width of the liquid sheet with diameter difference coefficient $\varepsilon$ for four different Reynolds number $R e_{j}$

It can be observed in Figure 4 (a) and (b) that for the very low Reynolds number regime (multichain regime at $R e_{j}=81$ ), the length and width of the liquid sheet follow the same trend. For $R e_{j}=162$, the laminar liquid sheet becomes unstable at $\varepsilon=0.2$, and it becomes laminar again for higher Reynolds numbers (Figure 4 and Figure 3 (left)). For $R e_{j}=203$, the laminar liquid sheet becomes unstable at $\varepsilon=0.2$ and it becomes laminar again at $\varepsilon=0.6$. For $R e_{j}=243$, the liquid sheet becomes unstable from $\varepsilon=0.2$ to $\varepsilon=0.6$. This shows that by increasing the Reynolds number the sensitivity of the liquid sheet onto the instability increased while it is damped by increasing the diameter difference coefficient which is the cause of the instability at the first step.

The instability of the liquid sheet at $\varepsilon=0.2$ leads to decrease in the length and width of the liquid sheet and they either increase or stay at the same vicinity for higher diameter difference coefficients. But as can be observed in Figure 4, there are some exception points such as point 1 in Figure 4 (a). At this point, the significant increase in the length and width of the liquid sheet is due to the transition of the liquid sheet from an unstable liquid sheet to a stable liquid 
sheet known as the leaf shape liquid sheet. The liquid sheet of point 1 is compared with the liquid sheet of point 2 in Figure 4 showing the same length and width of the liquid sheet with a lower $R e_{j}$ but in a stable regime.

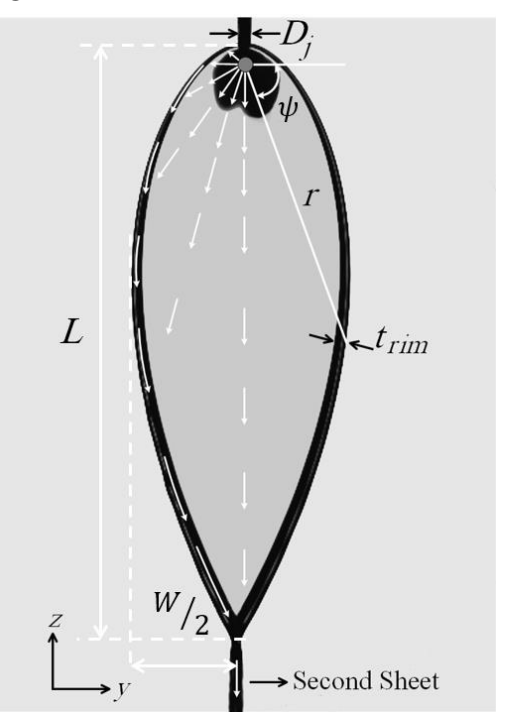

Figure 5. Image of the liquid sheet showing different parameters used to investigate the morphology of the liquid sheet

The liquid sheet produced by impinging two jets with the same velocity, $V_{j 1}=V_{j 2}$ for $\varepsilon=0,0.2$, 0.4 , and 0.6 and for Reynolds number of $81 \leq R e_{j} \leq 243$ is shown in Figure 3 (right). The most important point which can be observed from this figure is that all the liquid sheets are stable. Though, the morphology of the liquid sheet seems to change with changing the diameter difference coefficient $\varepsilon$.

Figure 6 (a) shows the variation of the non-dimensional radius of the liquid sheet, $r / D_{j}$ with the azimuthal angle $\psi$ for Reynolds number of $R e_{j}=203$. It can be observed that for all different diameter difference coefficient, the radius of liquid sheets follows the same trend, but the slope of each line of the plot shows that for higher value of $\psi$. The increase of the radius is higher which affect the bottom shape of the liquid sheet. The general increase in the radius of the liquid sheet increased by the diameter difference coefficient, $\varepsilon$ could be due to the higher flow rate of the second jets.

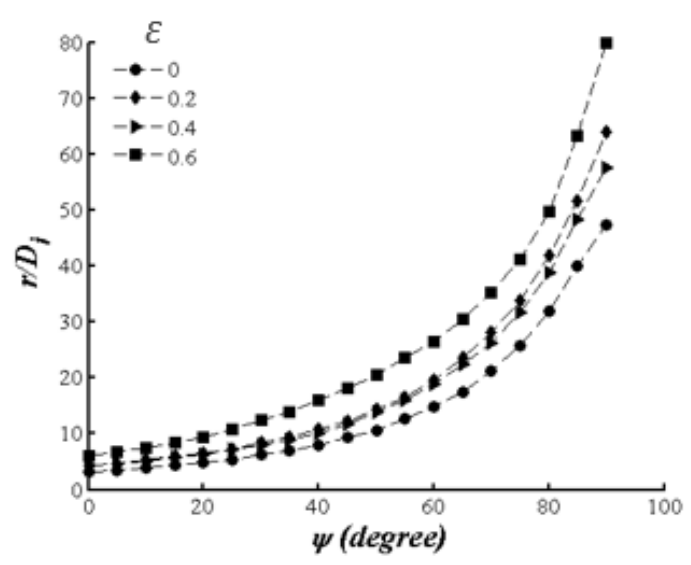

(a)

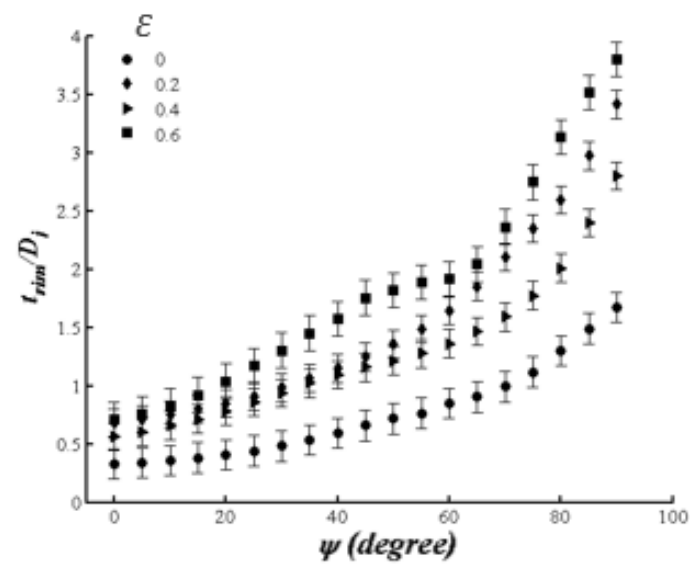

(b)

Figure 6. (a) Variation of the radius of the liquid sheet with the azimuthal angle for four different diameter difference coefficient $\varepsilon$ and $R e_{j}=203$. (b) Variation of the liquid rim thickness with azimuthal angle for four different diameter difference coefficient $\varepsilon$ and $R e_{j}=203$ 
Figure 6 (b) shows the variations of the non-dimensional liquid rim thickness of the liquid sheet, $t_{\text {rim }} / D_{j}$ with the azimuthal angle $\psi$ for Reynolds number of $R e_{j}=203$. As can be inferred from this figure, the liquid rim thickness increase by increasing the diameter difference coefficient $\varepsilon$ which could be due to the increase in the flow rate of the second jets. The increase in the liquid rim thickness by increasing the azimuthal angle $\psi$ is occurring for all four diameter difference coefficient $\varepsilon$, but this increase is higher for higher $\psi$.

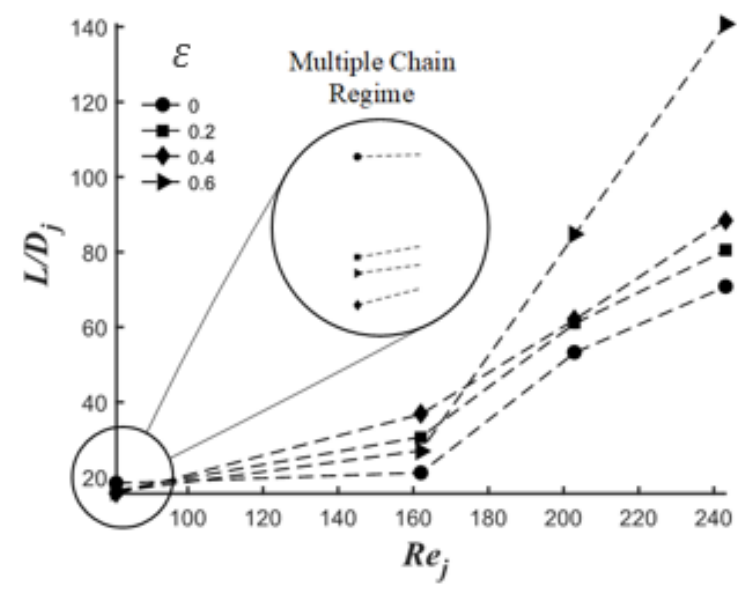

(a)

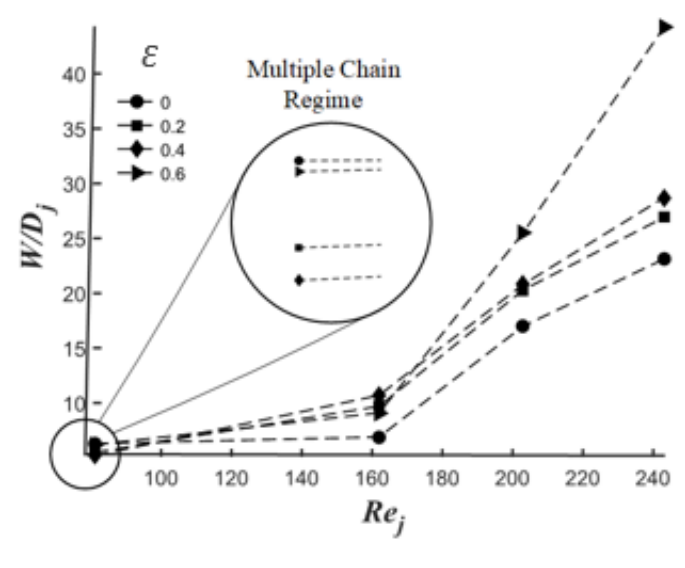

(b)

Figure 7. Variations of the (a) length and (b) width of the liquid sheet with Reynolds number $\operatorname{Re}_{j}$ for four different diameter difference coefficient $\varepsilon$

Variations of the non-dimensional length of the liquid sheet, $L / D_{j}$ and width of the liquid sheet $W / D_{j}$, with Reynolds number $R e_{j}=203$ and for all four diameter difference coefficient $\varepsilon$ are shown in Figure 7 respectively. For $R e_{j}>162$, the length of the liquid sheet is increasing by increasing the Reynolds number as well as the diameter difference coefficient $\varepsilon$. For multiple chain regime which occurs in the very low Reynolds numbers, it can be seen that a specific relation with the diameter difference coefficient cannot be observed though the liquid sheet length of $\varepsilon=0$ is significantly larger than the liquid sheet with diameter difference.

The width of the liquid sheet follows the same behaviour of the length. For $R e_{j}>162$ the width of the liquid sheet is increasing by increasing the Reynolds number as well as the diameter difference coefficient $\varepsilon$ but for the multiple chain regime, there is no relation between the diameter difference coefficient and the liquid sheet width.

\section{Conclusions}

Liquid sheet of two impinging jets with two different diameters is investigated for a low Reynolds number regime of the jets. The experimental investigations showed that the two imping jets with different jet diameter but with the same jets velocity form a stable leaf shape liquid sheet. In this case, the liquid rim thickness increase by increasing the diameter difference. The radius of the liquid sheet also increases by increasing the diameter difference in all azimuthal angles, though the behaviour of the maximum length and width of the liquid sheet are not same as the radius. It was also shown that two jets with the different diameter but with the same jets momentum can form both stable and unstable liquid sheet. It was shown that the maximum length and width of the liquid sheet both decrease by falling the liquid sheet to the unstable regime due to the diameter difference. Though, this behaviour could be different at the transient point from the unstable to the stable regime. 


\section{Acknowledgments}

The authors thank Arsin Tabesh Negran Fannavar Company for their sincere cooperation and the availability of PhotoFreezer v2.5. The financial support from the centre of research assistance of Sharif University of Technology (Grant No. G950411) is also gratefully acknowledged.

\section{References}

[1] A. Lefebvre and V. McDonell, Atomization and sprays. 2017.

[2] N. Ashgriz, Handbook of atomization and sprays: theory and applications. 2011.

[3] S. Y. Jejurkar, G. Yadav, and D. P. Mishra, "Characterization of impinging jet sprays of gelled propellants loaded with nanoparticles in the impact wave regime," Fuel, vol. 228, no. May 2017, pp. 10-22, 2018.

[4] A. J. Mahajan and D. J. Kirwan, "Micromixing effects in a two-impinging-jets precipitator," AlChE J., vol. 42, no. 7, pp. 1801-1814, Jul. 1996.

[5] S. Pal, K. Madane, and A. A. Kulkarni, Antisolvent based precipitation: Batch, capillary flow reactor and impinging jet reactor. Elsevier B.V., 2019.

[6] R. Baber, L. Mazzei, N. T. K. Thanh, and A. Gavriilidis, "Synthesis of silver nanoparticles using a microfluidic impinging jet reactor," J. Flow Chem., vol. 6, no. 3, pp. 268-278, 2016.

[7] K. Yamamura, J. Fukuzaki, Y. M.-C. engineering science, and undefined 2011, "Clathrate hydrate formation using liquid jets impinging on each other: An observational study using paired water jets or water and methylcyclohexane jets," Elsevier.

[8] M. Hafezi, M. Mozaffarian, M. Jafarikojour, M. Mohseni, and B. Dabir, "Application of impinging jet atomization in UV/H2O2 reactor operation: Design, evaluation, and optimization," J. Photochem. Photobiol. A Chem., p. 112198, Nov. 2019.

[9] Y. J. Choo and B. S. Kang, "The velocity distribution of the liquid sheet formed by two low-speed impinging jets," Phys. Fluids, vol. 14, no. 2, pp. 622-627, 2002.

[10] A. Kebriaee, S. Kashanj, and G. Olyaei, "Fishbone pattern formation due to asymmetries in colliding low-velocity jets," At. Sprays, vol. 28, no. 8, 2018.

[11] E. A. Ibrahim and A. J. Przekwas, "Impinging jets atomization," Cit. Phys. Fluids A Fluid Dyn., vol. 3, p. 2981, 1991.

[12] N. B. and E. Villermaux, "Atomization by jet impact," J. Fluid Mech, vol. 549, pp. 273-306, 2006.

[13] J. W. M. Bush, "On the collision of laminar jets" Artic. J. Fluid Mech., vol. 511, pp. 285-310, 2004.

[14] S. Kashanj and A. Kebriaee, "The effects of different jet velocities and axial misalignment on the liquid sheet of two colliding jets," Chem. Eng. Sci., vol. 206, 2019. 